Document downloaded from:

http://hdl.handle.net/10251/63936

This paper must be cited as:

Atkinson, J.; Glanc, M.; Prakorbjanya, M.; Sophocleous, M.; Sion, R.; Garcia-Breijo, E. (2013). Thick film screen printed environmental and chemical sensor array reference electrodes suitable for subterranean and subaqueous deployments. Microelectronics International. 30(2):92-98. doi:10.1108/13565361311314485

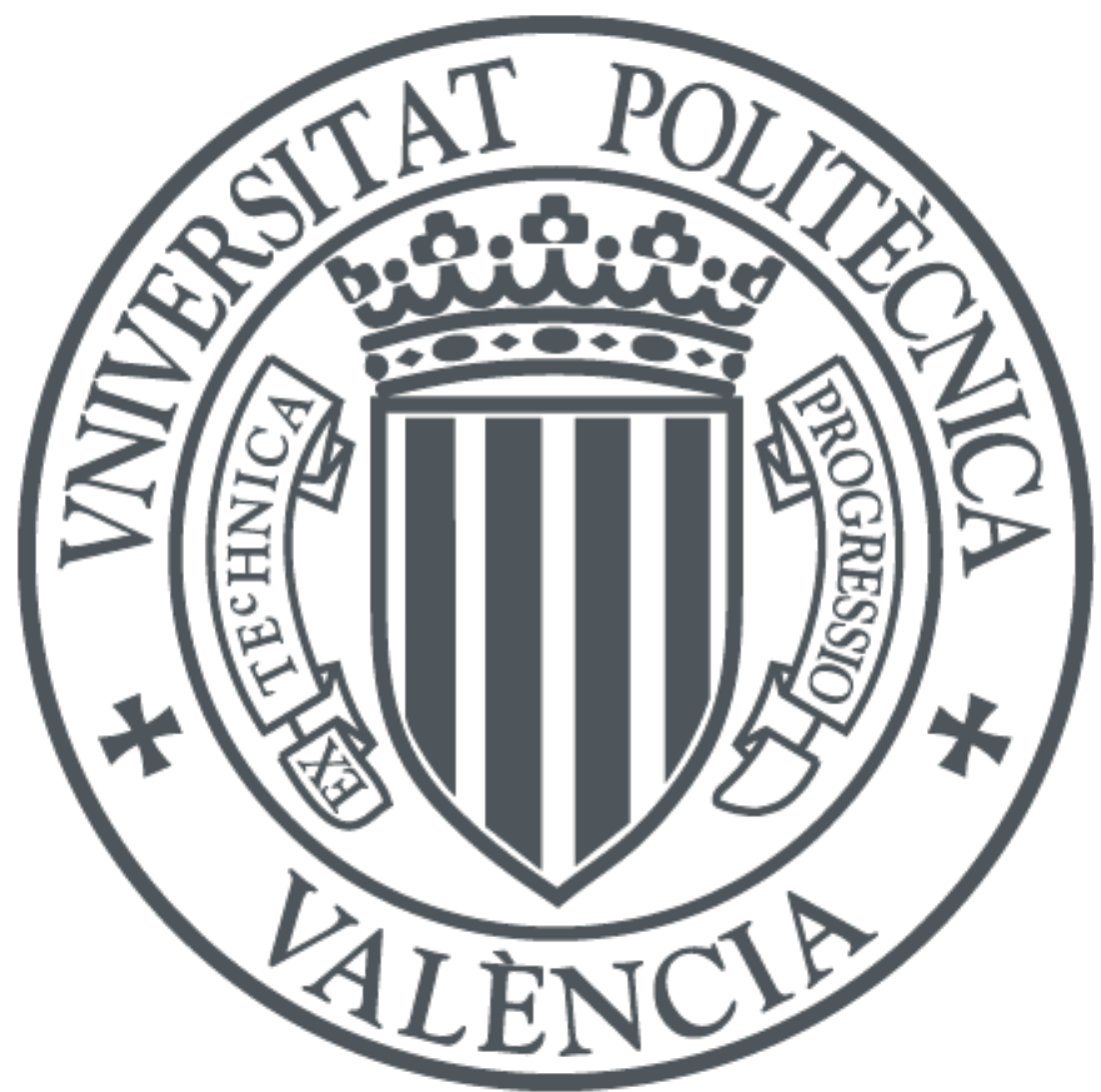

The final publication is available at

http://dx.doi.org/10.1108/13565361311314485]

Copyright Emerald

Additional Information 


\title{
Thick Film Screen Printed Environmental and Chemical Sensor Array Reference Electrodes Suitable for Subterranean and Subaqueous Deployments
}

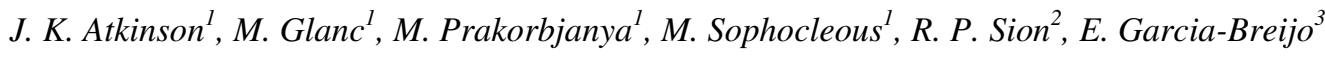 \\ ${ }^{I}$ Faculty of Engineering and the Environment, University of Southampton, United Kingdom \\ Email: jka@soton.ac.uk \\ ${ }^{2}$ C-Cubed Limited, Whitchurch, United Kingdom \\ ${ }^{3}$ Instituto de Reconocimiento Molecular y Desarrollo Tecnológico, \\ Universidad Politécnica de Valencia, Spain
}

\begin{abstract}
Thick film environmental and chemical sensor arrays designed for deployment in both subterranean and submerged aqueous applications are reported. Various choices of materials for reference electrodes employed in these different applications have been evaluated and the responses of the different sensor types are compared and discussed. Results indicate that the choice of binder materials is critical to the production of sensors capable of medium term deployment (e.g. several days) as the binders not only affect the tradeoff between hydration time and drift but also have a bearing on device sensitivity. Sensor calibration is shown to remain an issue with long term deployments (e.g. several weeks) but this can be ameliorated in the medium term with the use of novel device fabrication and packaging techniques.
\end{abstract}

\section{Introduction}

Screen printed (thick-film) fabrication methods are increasingly being used for the fabrication of chemical and environmental sensors. Arrays of these devices are easily colocated onto ceramic and other substrates for application in a variety of areas [1-3]. A photograph of a typical example of this type of device, a water quality sensor designed to be used for the simultaneous measurement of $\mathrm{pH}$, temperature, dissolved oxygen concentration, redox potential and conductivity, is shown in Figure 1 below.

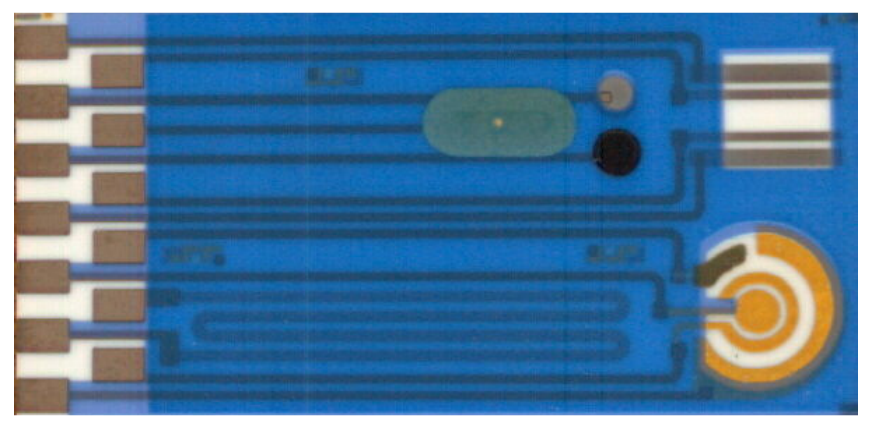

Fig. 1. Multi-element environmental sensor array comprising screen printed $\mathrm{pH}$, temperature, oxygen concentration, redox potential and conductivity sensors printed on a $25 \mathrm{~mm}$ x $50 \mathrm{~mm}$ alumina ceramic substrate

Deployment durations of these devices vary with their application and can be broadly classified as being short term, where calibration can be performed between samples (such as a hand held probe for example), medium term (several days) and long term (several weeks of undisturbed operation). As will be discussed here, due to their very nature, thick film chemical sensors are not generally well suited for unlimited operation. They, like most chemical sensors, suffer from electrode drift and require at best recalibration and sometimes complete replacement, depending on the type of sensor and the deployment environment. For example, leaching of functional materials (e.g. immobilized salts) will be faster into low ionic strength solutions than it will be in higher strength solutions. The work reported here concerns itself in particular with drift rates due to salt loss from electrodes and does not take account of other factors that limit deployment durations, such as bio-fouling, although this has been studied previously [1] and is the subject of a parallel programme of work.

Many of the physical sensors realised in thick film technology are well characterised and simply mimic their macro scale equivalents. Examples include platinum resistance thermometers and conductivity electrodes. Thick film chemical sensors however, often mimic electrochemical equivalents and inevitably require a stable electro-potential (reference electrode) in order to function. Examples include both potentiometric devices such as $\mathrm{pH}$, redox potential and other ion selective electrodes, and amperometric devices such as oxygen sensors and other fuel cells. Although the use of a conventional liquid electrolyte filled commercially obtained reference electrode is often employed in the characterisation of these devices, in order that they may be deployed in field applications they generally require a true solid state version. Various examples of these devices have employed a screen printed reference electrode, generally based on a silver/silver halide electrode. Sometimes the thick-film reference electrode takes the form of a pseudo-reference [5-7] whereby the silver/silver halide electrode surface is in direct contact with the analyte. As such the reference electrode function is based on knowledge of the environmental matrix into which it is deployed such that the stability of the electro-potential of the reference electrode is unimportant. In other instances the construction of the reference electrode attempts to mimic the operation of a conventional electrolyte-filled reference electrode usually through the immobilising of a halide salt reservoir on top of the reference electrode material [8-11]. These devices are designed to give absolute as opposed to relative electro-potential stability and are better suited to applications where chloride ion concentrations can vary widely, such as where brackish water is usually encountered in estuarine waters, tidal rivers etc.

The reference electrodes reported here are fabricated as screen-printed planar electrodes with a halide salt containing 
polymer layer deposited as the top layer of the electrode to give some measure of electro-potential stability in a similar manner to that of a conventional electrolyte filled reference electrode. Early attempts at fabricating electro-potentially stable devices concentrated on the use of a polymer bound potassium chloride filled layer that was screen printed onto a silver/silver chloride layer [12]

The types of devices described here have previously been employed successfully on chemical and environmental sensor arrays for use in a range of industrial application areas including $\mathrm{pH}$ control of power station water feed lines and aqueous printing inks [1]. In these cases the analyte matrix was relatively well understood and allowance could be made for electrode potential drift. However recent interest in the use of thick film chemical sensor arrays in longer term environmental monitoring applications in relatively unknown matrices requires that they have much better electro-potential stability than that previously attained.

\section{Typical electrode construction}

A typical thick-film electrode (in this case a reference electrode) is shown in Figure 2 below. Details of the component materials and fabrication procedure employed for this particular device have been documented previously [4]. Fabrication of individual array elements is typically achieved by the successive printing of layers of functional materials (e.g. metal oxide powder suspended in a polymer binder for a $\mathrm{pH}$ sensitive layer), metal conductors and waterproofing. An array of such sensors can be economically designed such that many of the individual layers of the sensors are fabricated simultaneously with those of their neighbours. For example many of the underlying conductor materials and overlying waterproofing materials are common to more than one of the sensing elements in the array.

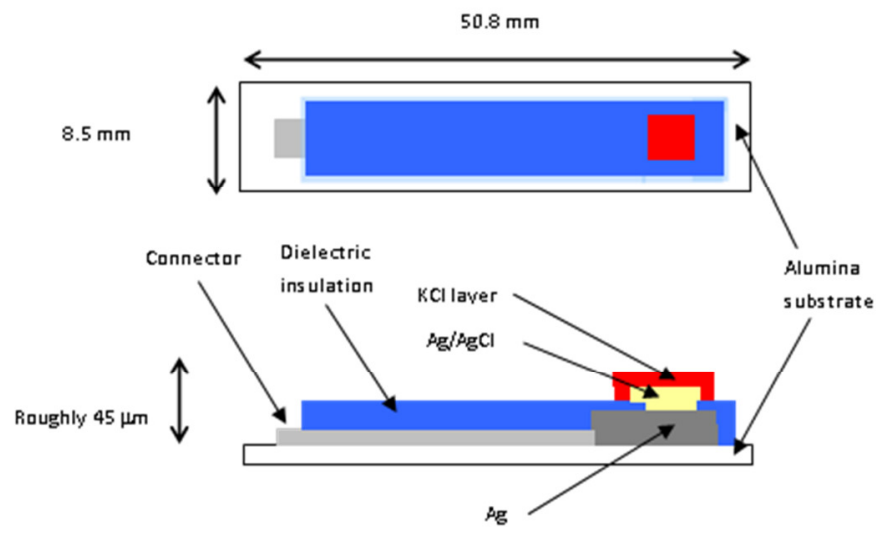

Fig. 2. Cross-sectional view of typical screen printed thickfilm electrode suitable for medium term deployment - in this case a silver/silver chloride reference electrode

\section{Electrode fabrication}

In order to produce sensor arrays suitable for medium to long term deployment, such as for subterranean applications for example, it is essential to control the electro-potential drift of the reference electrode. This stems from the requirement that calibration is not an available option on deployment, unlike the short term deployment strategies adopted for aqueous and other liquid sampling devices that are discussed in a later section.

Most thick film ion sensitive electrodes (e.g. pH) are found to exhibit reasonably stable potentials over the medium term but the design of a silver/silver chloride reference electrode conflicts with this requirement. This is due to the fact that in order to maintain a constant potential, the electrode requires a chloride salt containment layer of relatively high chloride ion concentration, at least as high as the maximum likely to be encountered in the given application. This high concentration of chloride ions then has a tendency to diffuse out of the salt containment matrix, usually a polymer binder, and into the analyte (e.g. surrounding soil). Attempts at minimising this drift rate are hampered by the need for the electrode to hydrate rapidly and then act as a good ionic conductor to give fast responding sensors when used in combination with an ion selective electrode for example. A reasonable compromise has been found to exist with polymer bound chloride salt concentrations of approximately $20 \%$ by weight [4].

The electrodes reported here were fabricated with an upper layer of ESL242 (modified silicone) containing $20 \%$ by weight of potassium chloride $(\mathrm{KCl})$. This upper layer of polymer bound $\mathrm{KCl}$ is in contact with an underlying silver/silver chloride layer that is fabricated on top of a silver back contact. In some cases the silver/silver chloride layer employed a commercially obtained polymer bound paste (GEM C61003P7) and in others a glass binder was used. The glass bound silver/silver chloride layers were fabricated from two screen printable pastes that were prepared as follows. Type 1 (Valencia PPCFC3) - AgCl (Aldrich 227927) milled to 400rpm/30min with ethanol (Pulverisette Fritsch), sieve $100 \mu \mathrm{m}$, Ag (Aldrich 32708-5) sieve $100 \mu \mathrm{m}$, powder frit (Ferro CF7567FC) sieve 100 $\mu \mathrm{m}$, vehicle (Heraeus V-006), mixed in the ratios $3 \mathrm{gr} \mathrm{Ag}+3 \mathrm{gr} \mathrm{AgCl}+2 \mathrm{gr}$ frit $+3.84 \mathrm{gr}$ vehicle and triple roll milled for 5 minutes. Type 2 (Valencia PPCFB2) is exactly the same as Type 1 with the exception of a different powder frit (Ferro EG2020VEG).

All the reference electrodes had a silver back contact constructed from ESL9912-A. All pastes were screened and fired according to their individual specifications with the exception of one batch of devices employing a polymer bound silver/silver chloride that was accidentally fired at 850 degrees Celsius (as opposed to the specified 150 degrees C) giving some very interesting results as reported below.

The metal oxide $\mathrm{pH}$ sensors are constructed in a very similar manner to the electrode shown in Figure 2 but with a polymer bound Ruthenium Oxide top layer (GEM C50502D7). In aqueous media these electrodes work on the principle of an equilibrated electro-potential response as illustrated by Equation 1.

$$
2 \mathrm{RuO}_{2}+\mathrm{H}_{2} \mathrm{O}+2 e^{-} \Leftrightarrow \mathrm{Ru}_{2} \mathrm{O}_{3}+2 \mathrm{OH}^{-} \quad \text { Eqn. } 1
$$

The $\mathrm{pH}$ electrodes have previously been found [13] to exhibit an approximately Nernstian response of $56 \mathrm{mV} / \mathrm{pH}$, albeit with small device to device offset potential differences that, depending on the accuracy requirement, may require calibration on deployment, using for example the method 
described in a following section dealing with the subject of sensor deployment.

\section{Electrode evaluation}

Electrode evaluations were undertaken through a series of experiments where the thick film reference electrode potentials were measured against a conventional commercially obtained reference electrode (Beckmann Coulter A57193) whilst being simultaneously immersed into various test solutions. The potential differences between the electrodes were recorded using a purpose constructed computer controlled multi-channel high input impedance analogue to digital converter unit. The test arrangement for the electrodes in solution is shown in Figure 3 below.

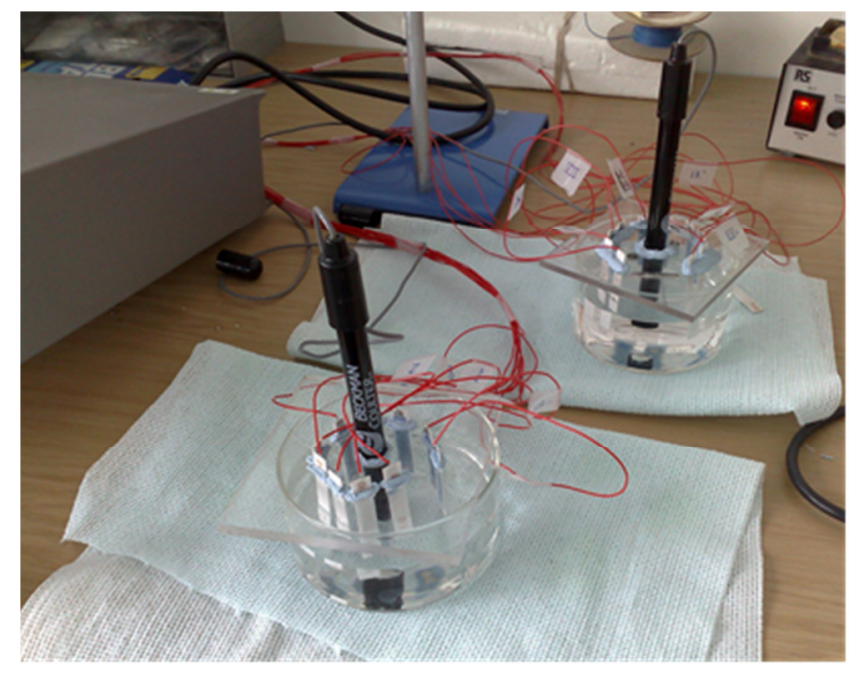

Fig. 3. Test set-up for thick-film reference electrode response testing through successive immersion in various test solutions.

The various test solutions included varying concentrations of dissolved $\mathrm{KCl}$ that were designed to produce decade step changes of chloride ion concentration, and $\mathrm{pH}$ buffer solutions giving step changes of $\mathrm{pH} 4, \mathrm{pH} 7$ and $\mathrm{pH} 10$. The decade step solutions of $\mathrm{KCl}$ were produced by the simple expedient of saturating deionised water with powdered $\mathrm{KCl}$ (approx. 3.5 Mol) and then successively diluting the solutions with deionised water in the ratio $9: 1$ to produce saturated solution ratios of 1:1, 1:10, 1:100 and 1:1000. The electrodes were then cycled through these various test fluids in groups in order to ascertain their sensitivity to chloride ion concentration change and their $\mathrm{pH}$ response. In the latter case groups of thick film $\mathrm{pH}$ electrodes were also tested simultaneously with the thick film reference electrodes.

\section{Experimental results}

\section{Testing of thick film reference electrodes in varying chloride ion concentrations}

Batches of thick film reference electrodes of varying construction were left to hydrate in a solution of saturated potassium chloride, diluted 1:100 with deionised water, for a period of approximately 12 hours and then subjected to cycling through test solutions of varying chloride ion concentration. The tested devices had different types of silver/silver chloride layer binder as described previously in the section detailing electrode construction. After two cycles of testing in varying chloride concentrations the devices were again left to soak in a solution of saturated potassium chloride diluted 1:100 with deionised water, this time for a 3 day "drift period", after which they were again cycled through the varying chloride concentrations. The entire sequence of tests is as illustrated in Figure 4 below.

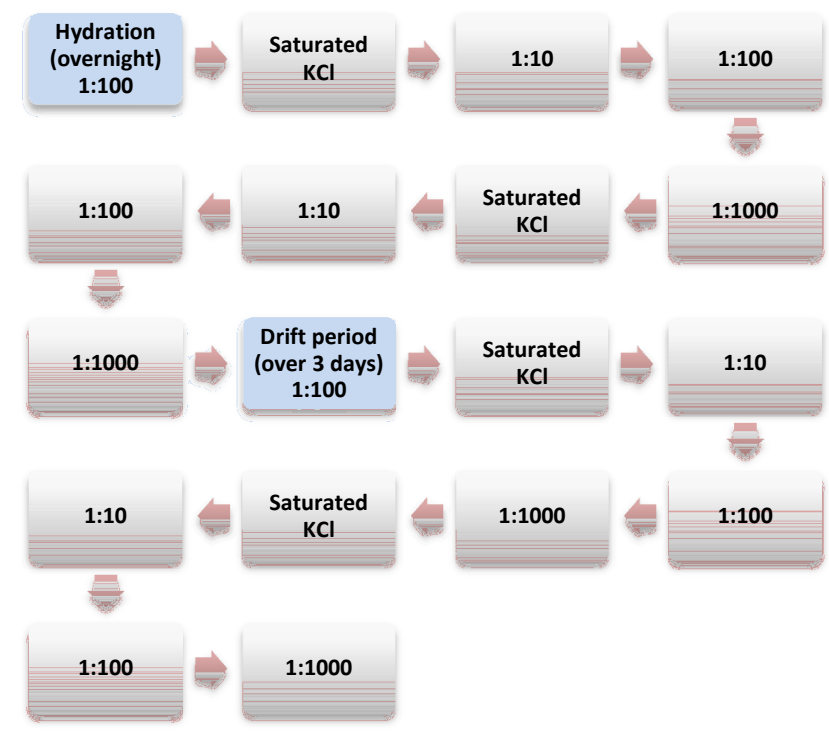

Fig. 4. Sequence for thick-film reference electrode response testing through successive immersion in various solutions.

The time history of the electrode potentials during this extended period of testing is as shown in Figure 5. The traces show the initial hydration period (denoted as A on the graph) and the step response of the electrodes as they are moved between the various test solutions during the testing intervals marked B and D. Figure 5 also shows a distinct drifting of some of the electrode potentials throughout the experiment and in particular during the 3 day interval marked as $C$. The bottom two traces that exhibit the greatest amount of electrode potential drift correspond to the electrodes that have no silver/silver chloride layer present.

Figure 6 shows the chloride sensitivity of the electrodes as a function of test solution concentration before this 3 day period of electrode potential drift (test interval B), while Figure 7 shows their chloride sensitivity after the 3 days (test interval D).

\section{Testing in pH buffers}

Figure 8 shows the response of thick-film $\mathrm{pH}$ electrodes (top traces) and thick-film reference electrodes (bottom traces) when the electrodes were cycled between $\mathrm{pH}$ buffer solutions

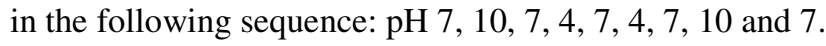

The silver/silver chloride reference electrodes show some $\mathrm{pH}$ sensitivity but this is very repeatable and manifests itself as a slight reduction in the overall sensitivity of the $\mathrm{pH}$ electrodes when they are paired with the thick-film reference electrodes. 


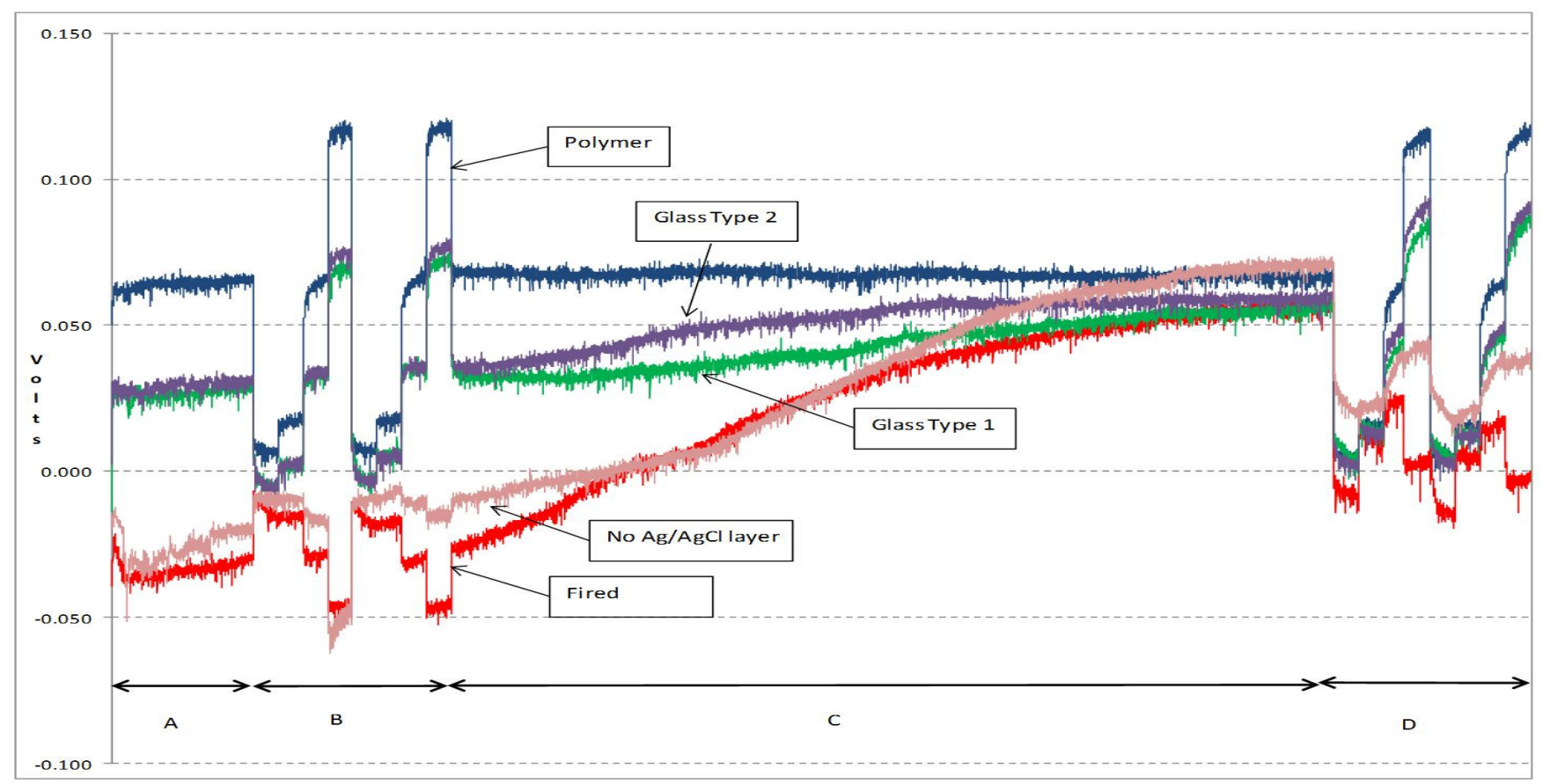

Fig. 5. Time history of reference electrode potentials (voltage relative to commercial reference electrode) during extended period of testing, with interval $\mathrm{C}$ corresponding to 3 days.

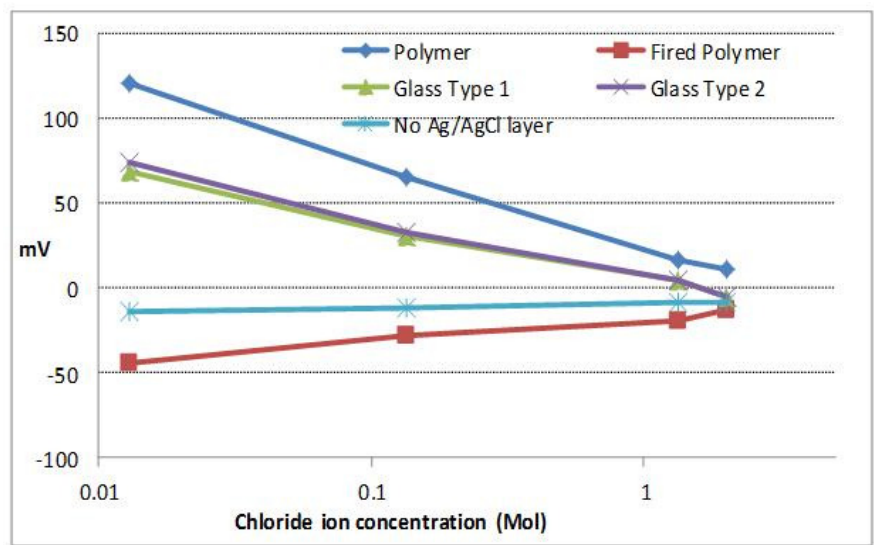

Fig. 6. Sensitivity of thick-film reference electrodes to chloride ion concentration during testing interval B (before 3 day drift period).

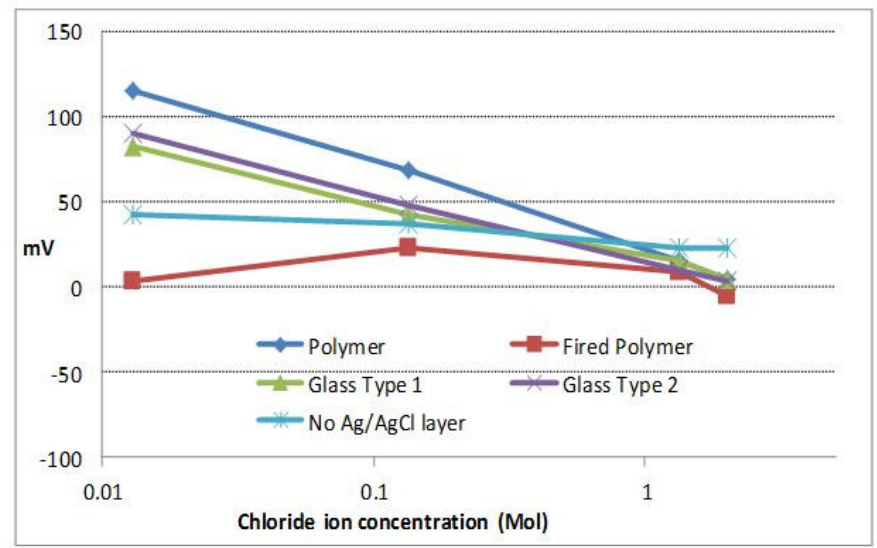

Fig. 7. Sensitivity of thick-film reference electrodes to chloride ion concentration during testing interval D (after 3 day drift period).

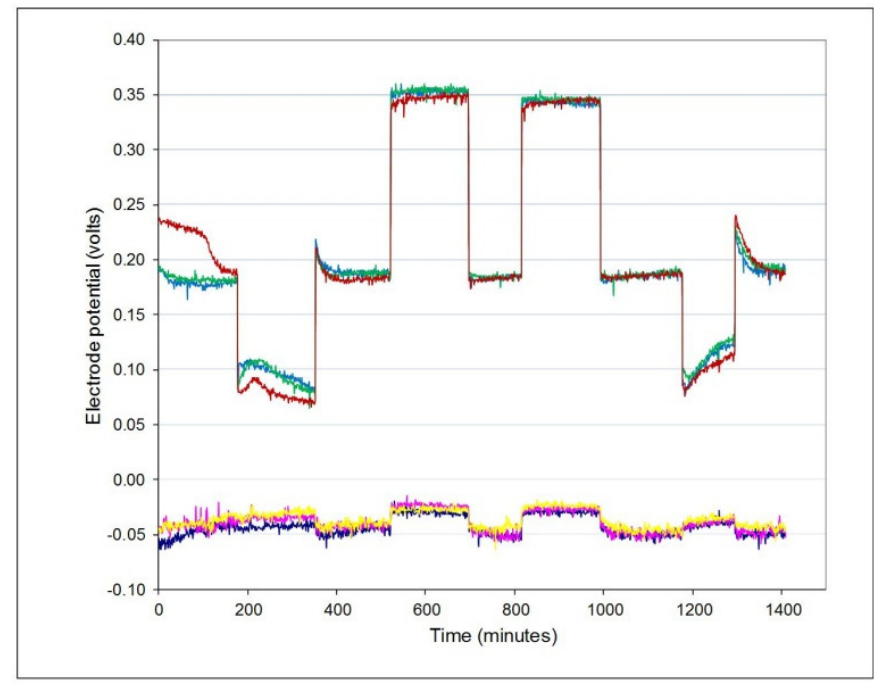

Fig. 8. Typical response of thick-film $\mathrm{pH}$ sensors (upper 3 traces) and reference electrodes (lower 3 traces) to successive immersions in $\mathrm{pH}$ buffer solutions in the sequence $\mathrm{pH} 7,10,7$, $4,7,4,7,10$ and 7.

\section{Discussion}

It is clear from the results that the reference electrodes with the polymer bound silver/silver chloride layer exhibited the greatest (least desirable) sensitivity to varying chloride concentration (approx. $50 \mathrm{mV} /$ decade $\left[\mathrm{Cl}^{-}\right]$), while the devices with no silver/silver chloride layer exhibited the most stable responses $\left(<10 \mathrm{mV} /\right.$ decade $\left.\left[\mathrm{Cl}^{-}\right]\right)$. This group included the devices where a polymer bound layer was accidentally fired at high temperature (850 degrees C), thereby effectively removing the silver/silver chloride layer from the surface of the silver back contact, a fact that was later established with 
the aid of electron microscopy. The sensitivity of both types of devices with glass bound silver/silver chloride layers lay in between these other two types (approx. 30mV/decade $\left[\mathrm{Cl}^{-}\right]$). The sensitivities (in $\mathrm{mV} /$ decade $\mathrm{Cl}^{-}$) of all electrode types, both before and after the 3 day drift test, are shown in Table 1 below.

\begin{tabular}{|c|c|c|}
\hline $\begin{array}{c}\text { Ag/AgCl layer } \\
\text { binder type }\end{array}$ & $\begin{array}{c}\mathbf{m V / d e c a d e ~} \mathbf{C l}^{-} \\
\text {before drift test }\end{array}$ & $\begin{array}{c}\mathbf{m V / d e c a d e ~} \mathbf{C l}^{-} \\
\text {after drift test }\end{array}$ \\
\hline Polymer bound & -47 & -49 \\
\hline Glass - Type 1 & -30 & -33 \\
\hline Glass - Type 2 & -33 & -38 \\
\hline Fired polymer & +12.6 & -7.6 \\
\hline None (bare Ag) & +2.5 & -9.6 \\
\hline
\end{tabular}

Table 1: Sensitivity of various different reference electrode types in $\mathrm{mV} /$ decade $\mathrm{Cl}^{-}$before and after 3 day drift testing

The best chloride ion stabilities were obtained with the electrodes that had no silver/silver chloride layer. However, these devices were also the most susceptible to longer term drift. From inspection of Figure 5 it can be seen that the potentials of the electrodes with no silver/silver chloride layer drifted from an initial potential of approximately $-25 \mathrm{mV}$ w.r.t. the commercially obtained liquid electrolyte reference electrode, to a final potential of approximately $+60 \mathrm{mV}$. Likewise the glass bound silver/silver chloride devices drifted from an initial potential difference of approximately $+30 \mathrm{mV}$ w.r.t. the commercial liquid electrolyte reference electrode to a similar final value of approximately $+60 \mathrm{mV}$.

What is also very interesting is the change in the slope of the response of the devices with no silver/silver chloride layer, from positive with respect to increasing chloride ion concentration before the 3 day drift period, to negative with respect to increasing chloride ion concentration after the 3 day drift period. This, coupled with the converging potentials just described, would tend to suggest that the initially bare silver back contact of these electrodes has chloridised during the interim 3 day period. This result also appears to indicate that the initially bare silver electrode, with a covering layer of $20 \%$ potassium chloride in polymer binder, has a different response mechanism compared to the silver/silver chloride layer devices.

An apparent anomaly in the results appears with the response of the accidently fired polymer silver/silver chloride sensors. The response to 1:1000 dilution of saturated potassium chloride solution after the 3 day drift period, as shown in Figure 7, does not follow the trend of these electrodes before the 3 day drift period (Figure 6), or indeed any of the other devices, increasing at first positive with increasing chloride concentration and then becoming more negative beyond approx. $0.1 \mathrm{M}$. It is tempting to think this may have been the result of experimental error, however this response was in fact very repeatable over a series of experiments and can be seen in the traces shown in Figure 5, during test interval D. This behaviour of the fired polymer silver electrodes merits further investigation and is believed possibly to be the result of localised changes in the silver/silver chloride ratio within the surface layers of the thick film devices as explained below.
The potential response of the silver/silver chloride electrode is governed by the equilibrium reaction:

$$
\operatorname{AgCl}_{(s)}+e^{-} \leftrightarrow A g_{(s)}+C l_{(a q)}^{-} \quad \text { Eqn. } 2 .
$$

Hence the equilibrium potential (E) of the electrode is given by the Nernst equation as:

$$
E=E^{\theta}+\left(\frac{R T}{n F}\right) \ln \frac{(O x i)}{(R e d)} \quad \text { Eqn. } 3 .
$$

Where $E^{\theta}$ is the standard potential (V); $R$ is the universal gas constant $\left(8.314 \mathrm{JK}^{-1} \mathrm{~mol}^{-1}\right) ; T$ is the absolute temperature $(\mathrm{K}) ; n$ is the number of moles of electrons transferred in the reaction; $F$ is the Faraday constant $\left(96485 \mathrm{C} \mathrm{mol}^{-1}\right)$; Oxi is the activity of the oxidant and Red the activity of the reductants.

If the activities of the oxidant and reductants are assumed to be approximately equal to their concentrations the electrode potential is then given by:

$$
E=E^{\theta}+\left(\frac{R T}{n F}\right) \ln \frac{[\mathrm{AgCl}]}{[\mathrm{Ag}]\left[\mathrm{Cl}^{-}\right]}
$$

Eqn. 4.

However, the Nernst equation for a cell is more frequently expressed in terms of base 10 logarithms at approximate room temperature (assumed to be $25^{\circ} \mathrm{C}$ ), in which case Eqn. 4 can be re-written as:

$$
E=E^{\theta}+0.0592 \frac{[\mathrm{AgCl}]}{[\mathrm{Ag}]\left[\mathrm{Cl}^{-}\right]}
$$

Eqn. 5.

By separating out the dissolved from the solid components Eqn. 5 can then be re-written as:

$$
E=E^{\theta}-0.0592 \log \left[\mathrm{Cl}^{-}\right]+0.0592(k) \quad \text { Eqn. } 6 .
$$

Where, $(k)$ is the ratio of the concentration of silver chloride to silver, which is generally assumed to be approximately constant. Hence, if at room temperature the measured electrode potential $(E)$ decreases by approximately $59 \mathrm{mV}$ for every decade increase in chloride ion concentration, the electrode can be said to display Nernstian behaviour.

In most studies of thick-film electrodes, the relative concentrations of silver chloride and silver are generally held to be constant given the quantities of material used in the device fabrication. However at the electrode surface this is not necessarily the case as the partially soluble silver chloride can itself dissolve into solution at the same time as a silver chloride layer can also be grown onto any exposed silver particles by chemical reaction, which is particularly the case if the immersion solution contains any significant concentration of chloride ions. This fluctuation in the silver chloride and silver concentrations can result in changes in their ratio $(k)$, which in turn affects the potential of the electrode, as demonstrated by Eqn. 5. Hence the electrode potential is not simply a function of chloride ion concentration, as assumed by Eqn. 6, but is in fact a more complex equilibrium determined by the solution concentration of chloride ions, the electrode surface concentrations of silver and silver chloride and the ease with which the silver chloride is able to dissolve and re- 
form, as determined by the binder type for example. It is believed that this mechanism may well account for the behaviour of the thick-film electrode types investigated here.

Although the devices were covered by a polymer bound chloride salt layer containing $20 \% \mathrm{KCl}$ by weight, it is understood from earlier work [4] that this polymer layer rapidly loses its salt on immersion in solution through dissolution of the $\mathrm{KCl}$, leaving behind a porous layer whose chloride ion concentration approximately corresponds to that of the immersing solution. Consequently, while any exposed silver at the electrode surface is increasingly likely to be chloridised in increasing chloride ion concentration solutions to give an increasingly positive potential, as per Eqn. 5, there is also the likelihood of increasing dissolution of silver chloride into lower concentration chloride ion solutions to give a subsequently negative change in electrode potential.

In the case of the initially non-silver chloride containing electrode types (bare silver and fired polymer), the overlying polymer bound $\mathrm{KCl}$ salt matrix appears to provide a source of chloride ions that enables an initial growth of a thin layer of silver chloride on the electrode surface. The subsequent porosity of the $\mathrm{KCl}$ salt matrix layer does not provide an adequate buffer to maintain a relatively stable chloride ion concentration however, as the dissolution and chloridisation of the thin silver chloride layer appear to be controlled to a large extent by the chloride ion concentration of the immersing solution. Hence increased dissolution of silver chloride in increasingly lower chloride ion concentration solutions gives rise to a negative change in electrode potentials, as per Eqn. 5, which appears to counterbalance the Nernstian chloride ion response of the thinly formed layer of silver chloride.

In contrast, the silver/silver chloride containing electrodes also experience a negative Nernstian change in potential with increasing chloride ion concentration as per Eqn.6, but do not appear to experience any appreciable change in their silver chloride to silver ratio as a result of increased dissolution in low ionic strength solutions. This is believed to be because the ratio $k$ in Eqn. 5 is made up from much larger amounts of silver chloride than in the case of the initially bare silver electrodes. Hence the effect of changes in the surface silver chloride concentration on this ratio is substantially lower and has less effect on the overall chloride ion response of the electrodes. However, the degree to which these particular electrodes respond, i.e. their sensitivity or slope, is apparently influenced by the binder type.

The polymer bound silver chloride devices exhibited a near Nernstian slope of $50 \mathrm{mV}$ per decade chloride ion concentration, compared with the glass bound silver chloride devices which exhibited a slope of approx. $30 \mathrm{mV}$ per decade chloride ion concentration. This observation merits further investigation of the device silver/silver chloride layer morphologies, but an initial consideration reveals that the polymer bound devices have a much thicker silver/silver chloride layer of approximately 50-80 microns, compared to their fired glass bound counterparts, which have thicknesses of typically 10-20 microns. It is likely that these differences in layer binder thickness account for the differences in the response due to the increased inhibition of silver chloride ion dissolution in the thick, i.e. less porous polymer devices than their thinner glass bound counterparts. Hence the Nernstian response of the glass bound silver/silver chloride layer devices is counteracted to some extent in lower ionic strength solutions in the higher porosity silver/silver chloride layer devices. Consequently, the higher the porosity of the layer, the more stable was the response of the electrodes, over the range of concentrations tested.

\section{Sensor array deployment}

The work reported here derives largely from two projects concerning the application of thick-film chemical and environmental sensor arrays in water and soil sensing. One project (FUSE) involves the deployment of the devices in a floodplain underground sensor network [15], while the other project, part funded by the UK Environment Agency, seeks to deploy the sensors for water catchment area monitoring. Both these work programs require the use of a combination of remote wireless sensing and hands portable manual testing using a variety of sensing devices.

A key aspect of all these applications however is the requirement to calibrate the sensors before use and preferably at the point of deployment. This is more easily achieved with hands portable devices and one approach to this is illustrated in Figure 9 below whereby a thick film sensor array is mounted in a probe assembly via a waterproofed connector. Behind the waterproof connector are the interface circuits that enable the sensors to be connected, either wired or wirelessly, to a portable personal computer for example, or other suitable form of data acquisition. The sensor array is protected in a cup-like container that screws onto the bottom of the probe assembly and contains holes through which the analyte (e.g. river water) can pass. A further bottom container containing a known calibration matrix (e.g. having known $\mathrm{pH}$ and conductivity values) is then screwed over and onto the protective cup. Deployment then consists of taking an initial reading of sensor values in the known calibration matrix to establish baseline readings (e.g. electrode offset potentials) prior to removal of the bottom container and deployment of the sensor in the analyte.

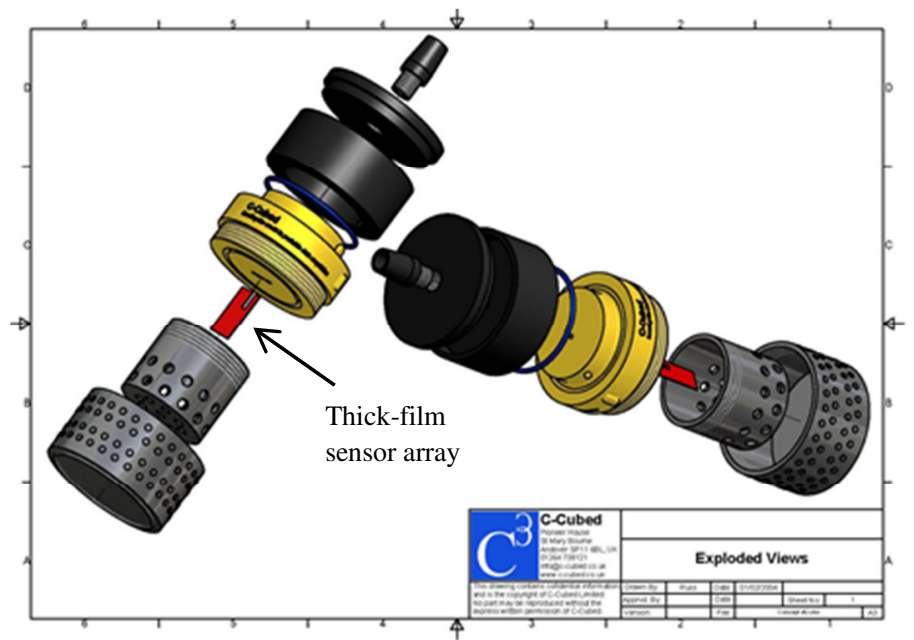

Fig. 9. Typical deployment of thick-film sensor array (red) in immersion probe assembly

However, sensors for use in longer term deployments, such as subterranean sensing, do not offer the luxury of this ease of 
calibration on deployment. Consequently it is important that the reference electrodes used in these applications are able to operate throughout their useful lifetimes without recalibration. Hence in this context, it is generally more important to minimize electrode drift, possibly at the expense of increased chloride ion sensitivity.

\section{Conclusions}

It can be concluded from the results reported here that through the careful choice of materials it is possible to fabricate thick film electrochemical sensors that are suitable for a variety of different application areas. For example, for short term deployments in water where chloride ion concentrations are liable to wide variation, it may be preferable to employ a reference electrode with a bare silver back contact (no silver/silver chloride layer) and trade off a higher rate of drift for an increased stability (insensitivity) to chloride ion concentration variation.

However, for longer term deployment, in soil sensing for example, it would appear preferable to employ a device with a thicker silver/silver chloride layer, resulting in an increased sensitivity to chloride ion concentration variation but a lower longer term potential drift. For soil sensing in particular this tradeoff may well prove acceptable given that chloride concentration changes may not be so wide ranging as in a purely aqueous environment. In addition, it has been shown to be possible to utilise a bare silver/silver chloride electrode to track chloride ion concentrations in soil [16] and hence it may be possible to compensate for an unwanted reference electrode chloride ion response in this manner.

\section{Acknowledgments}

The University of Southampton based authors gratefully acknowledge funding from the UK Environment Agency and the UK National Environmental Research Council (NERC).

\section{References}

1. Gac, A., Atkinson, J.K., Zhang, Z. and Sion, R.P. (2002) A comparison of thick-film chemical sensor characteristics in laboratory and on-line industrial process applications, Measurement Science and Technology, 13, 2062-2073.

2. Glasspool, W.V. and Atkinson, J.K. (2003) An evaluation of the characteristics of membrane materials suitable for the batch fabrication of dissolved oxygen sensors, Microelectronics International, 20, (2), 32-40.

3. Gac, A., Atkinson, J.K., Zhang, Z., Sexton, C.J., Lewis, S.M., Please, C.P. and Sion, R.P., (2004) Investigation of the fabrication parameters of thick film titanium oxidePVC $\mathrm{pH}$ electrodes using experimental designs, Microelectronics International, 21/3, 44-53.

4. Atkinson, J.K., Glanc, M., Boltryk, P., Sophocleous, M. and Garcia-Breijo, E., (2011) An investigation into the effect of fabrication parameter variation on the characteristics of screen-printed thick-film silver/silver chloride reference electrodes, Microelectronics International, 28/2, 49-52.

5. Andreescu, S., Noguer, T., Magearu, V. and Marty, J-L. (2002) Screen-printed electrode based on AChE for the detection of pesticides in presence of organic solvents, Talanta 57 169-176.
6. Boujtita, M., Hart, J.P. and Pittson, R. (2000) Development of a disposable ethanol biosensor based on a chemically modified screen-printed electrode coated with alcohol oxidase for the analysis of beer, Biosensors \& Bioelectronics 15 257-263

7. Matsumoto, T., Ohashi, A. and Ito, N. (2002) Development of a micro-planar $\mathrm{Ag} / \mathrm{AgCl}$ quasi-reference electrode with long-term stability for an amperometric glucose sensor, Analytica Chimica Acta 462 253-259

8. Mroz, A., Borchardt, M., Diekmann, C., Cammann, K., Knoll, M. and Dumschat, C. (1998) Disposable reference electrode, Analyst, Vol. 123 (1373-1376)

9. Simonis, A., Lüth, H., Wang J. and Schöning, M.J. (2004) New concepts of miniaturised reference electrodes in silicon technologyfor potentiometric sensor systems, Sensors and Actuators B 103 429-435

10. Tymeckia, Ł., Zwierkowskab, E. and Koncki, R. (2004) Screen-printed reference electrodes for potentiometric measurements, Analytica Chimica Acta 526 3-11

11. Maminska, R., Dybko, A. and Wroblewski, W. (2006) Allsolid-state miniaturised planar reference electrodes based on ionic liquids, Sensors and Actuators B, 115 552-557

12. Cranny, A.W.J. and Atkinson, J.K. (1998) Thick film silver-silver chloride reference electrodes, Measurement Science and Technology, 9, (9), 1557-1565.

13. Mihell, J.A. and Atkinson, J.K. (1998) Planar thick-film $\mathrm{pH}$ electrodes based on ruthenium dioxide hydrate, Sensors and Actuators B: Chemical, 48, (1-3), 505-511.

14. Atkinson, J.K., Cranny, A.W.J., Glasspool, W.V. and Mihell, J.A., An investigation of the performance characteristics and operational lifetimes of multi-element thick film sensor arrays used in the determination of water quality parameters, Sensors and Actuators B: Chemical, 54, 3, pp 215-231, 1999

15. Floodplain Underground Sensors Network (FUSE) project website is at http://www.fuseproject.org.uk/about

16. Cranny, A.W.J., Harris, N. R., Nie, M., Wharton, J. A., Wood, R. J. K. and Stokes, K. R. (2011) Screen-printed potentiometric $\mathrm{Ag} / \mathrm{AgCl}$ chloride sensors: Lifetime performance and their use in soil salt measurements. Sensors and Actuators A: Physical, 169 (2). pp. 288-294. 